\title{
Staphylococcus and Linezolid Resistance in Iran
}

\section{Fariba Shirvani, ${ }^{1}$ Anahita Sannai Dashti, ${ }^{2}$ and Kimia Seifi ${ }^{3,}{ }^{*}$}

${ }^{1}$ Pediatric Infections Research Center, Research Institute For Children Health, Shahid Beheshti University of Medical Sciences, Tehran, Iran

${ }^{2}$ Pediatrician, Subspecialist in Pediatric Infectious Diseases, Associate Professor, Ostad Alborzi Research Centre, Namazi Hospital, Shiraz Medical University, Shiraz, Iran

${ }^{3}$ Young Researchers and Elite Club, Roudehen Branch, Islamic Azad University, Roudehen, Iran

"Corresponding author: Kimia Seifi, Young Researchers and Elite Club, Roudehen Branch, Islamic Azad University, Roudehen, Iran. Fax: +98-2122226941, E-mail: kimiaseifi@yahoo.com

Received 2017 April 29; Revised 2017 July 10; Accepted 2017 December 25.

Keywords: Staphylococcus, Resistance, Iran

\section{Dear Editor,}

In traditional human communities, before introduction of antimicrobial agents, Staphylococcus aureus bacteremia fatality rate was $90 \%$. Soon after penicillin introduction, resistance to this agent was noticed during 1942 (1) and, it was increased to around 14\% in 1945 (2), and 82\% to $84 \%$ in 1967 (3). There has been a similar trend in Iran. According to a meta-analysis study on staphylococcus resistance patterns in Iranian hospitals during year 2012 (Askari et al.), the mean total prevalence of MRSA was $52.7 \% \pm 4.7$, and more than $50 \%$ in many Iranian cities. This means, treatment of more than half of $S$. aureus infections in Iran may be faced with difficulty. Noted prevalence of MRSA in Iran is moderately higher than Australia and lower than the United States (4). This lower report may be because of a shortage of diagnostic tools in the laboratories and lack of information. In a study conducted on $726 \mathrm{~S}$. aureus isolates (2007 to 2011) from 3 referral hospitals in Tehran (Iran), 30\% of strains were found to be MRSA and mec A gene was detected among all MRSA isolates. No strains were resistant to vancomycin, synercid, linezolid, and chloramphenicol (5). In a random sample population from 4 hospitals including various clinical samples in Tabriz from year 2005 to 2012, 151 Staphylococcus aureus isolates were separated and meca gene, representative of MRSA was found in 35\%, and $95 \%$ were resistant to penicillin and all isolates were susceptible to vancomycin and teicoplanin yet 5.9\% were resistant to linezolid (6). In a study on clinical isolates of 22 Staphylococcus strains from 22 admitted children ( 2 to 11 months) in Logman hospital (2012 to 2013), 5 isolates were methicillin resistant and all isolates were susceptible to linezolid and vancomycin; the AGR group 1 gene had the highest rate with increase in the capability of the organism to make biofilms (7).

Prevalence of ica ADBC genes was relatively high among these isolates and also all 4 ica genes were detected among MRSA strains. The presence of this gene increased biofilm formation (8).

Staphylococcal nasal carriage from 600 HCWs (2006; Namazi hospital/Shiraz) indicated methicillin-sensitive $S$. aureus (MSSA) in $25.7 \%$ and MRSA in 5.3\%, with the highest nasal carriage of MRSA in surgical wards and the emergency department. None of the MRSA strains were resistant to mupirocin, linezolid, fusidic acid, or vancomycin (9). In a study on 238 S. aureus-isolated clinical samples from different wards of Imam Reza hospital (Mashhad; 2011 to 2012), 5.88\% were resistant to quinupristindalfopristin, 5.46\% to linezolid, and 5.04\% to vancomycin. Vancomycin, a suitable antibiotic against this pathogen has a high price and recent emergence of vancomycin resistance in Iran has become noticeable and alarming (10). Nasal samples from 229 health care providers (Mofid children's hospital; 2013) were positive for staphylococcus in 27 cases while 21 were MRSA. All isolates were susceptible to linezolid and vancomycin; 2 isolates (9\%) had SCCmec I that has higher prevalence in HA-MRSA (11).

It is important to mention that increasing rectal colonization with VRE-positive for van A and van $B$ genes in children admitted to the PICU has a potential danger of transmission to gram positive organisms, such as Staphylococcus aureus (12).

Identification of methicillin resistance in Staphylococcus species (MRSA) is demonstrated by resistance to oxacillin and cefoxitin with the disk diffusion method (Kirby-Bauer) $(13,14)$.

Minimum inhibitory concentration (MIC) is determined by the microbroth dilution method and the polymerase chain reaction (PCR) Method is done for detection of mecA genes (15). If MRSA is detected, its resistance to available antibiotics such as clindamycin aminoglyco- 
sides, cotrimoxazole, vancomycin, and linezolid should be investigated.

In the recent years, gene studies showed the characteristics, detailed antibiotic resistance, and source of genetic information of microorganisms. In a study on linezolid resistance in hospital-acquired microorganisms (Shanghai; 2014), from 2 teaching hospitals, 17 Staphylococcus strains with linezolid-resistance were found. Fourteen were Staphylococcus capitis, and 3 were Staphylococcus aureus. Staphylococcus capitis isolates had C316T (Arg106Cys) substitution carried on protein L4 and/or had the cfr gene. Linezolid-resistant S. aureus strains had C389G (Ala130Gly) substitution carried on protein L3, and/or had the cfr gene (16).

In an Iranian report of linezolid resistance, Armin studied 631 anterior nare point-prevalence of S. aureus colonization in children admitted for 48 hours or more in different wards of Mofid Children's Hospital. Staphylococcus aureus and Methicillin Resistant Staphylococcus aureus (MRSA) colonization rate was $3.2 \%$ and $1.1 \%$, respectively.

Antibiotic susceptibility pattern was determined by Minimum Inhibitory Concentration (MIC), according to CDC guidelines of the 2011 Clinical and Laboratory Standards Institute (CLSI). From 20 positive cases of Staphylococcus aureus, 13 were MSSA and 7 cases were MRSA. All MRSA isolates were susceptible to rifampin and clindamycin. Six cases of MRSA and 7 cases of MSSA were resistant to vancomycin. Resistance to linezolid was detected in 19 from 20 Staphylococcal isolates, and 15 from 19 linezolid resistant strains were found to be positive for chloramphenicol florfenicol resistant gene (cfr gene) (14).

There are very few reports of vancomycin and linezolid resistant MRSA in Iran $(4,5,17)$; Fathollahzadeh $(2004$ to 2005; Tehran) reported that 99 out of 277 (36\%) hospitaladmitted wound or blood specimens were MRSA Staphylococcus aureus isolates. All were susceptible to vancomycin, teicoplanin, linezolid, and tigecycline. At least $95 \%$ of them were also resistant to ciprofloxacin, erythromycin, kanamycin, azithromycin, tetracycline, and gentamicin (18).

In a study on 238 clinical samples, collected from different wards of Imam Reza hospital of Mashhad (2011 to 2012), $5.46 \%$ of cases of MRSA were resistant to linezolid (10).

Armins study showed a high resistance to linezolid and vancomycin with concomitant susceptibility to rifampin and clindamycin, which shows the probability of specific gene expression in these strains. The MIC report in the range of 8 to $32 \mu \mathrm{g} / \mathrm{mL}$, in linezolid resistant Staphylococcus strains may show false negative results in the first 24 hours of incubation.

In a study on specific linezolid resistant MRSA (MRSA CM-05), an automated method (Vitek system; BioM'erieux,
Marcy l'Etoile, France) detected MIC of $16 \mu \mathrm{g} / \mathrm{mL}$, initially. Both the agar dilution methods and broth results were similar. The E test method reported MIC of $2 \mu \mathrm{g} / \mathrm{mL}$ after 24 hours of incubation, yet after incubation of 48 hours, a second halo of inhibition with an MIC of $16 \mu \mathrm{g} / \mathrm{mL}$ was seen. The results indicated that the $\mathrm{E}$ test in cfr-mediated linezolid might not detect resistance with standard procedures and a longer time of incubation may be needed. Another explanation is that with respect to the appearance of a double zone of inhibition by the E test, within MRSA CM05, a "heterogeneous" subpopulation of linezolid-resistant cells is possible, this may occur because of a common feature of MRSA, which is differential expression of resistance genes. This clinical isolate of MRSA with human source showed linezolid-mediated resistance by the $\mathrm{cfr}$ gene, which encodes a $23 \mathrm{~S}$ rRNA methyltransferase. This resistance pattern occurred in a country with very limited usage of linezolid. This type of MRSA resistance is still very rare, but clinicians should be aware of the possibility of dissemination from animals to humans by horizontal gene transfer, which could occur between enterococcal and staphylococcal isolates of both human and animal origin (19). Armin's study did not report the MIC of Staphylococcus strains, which is important to be reported because of this critical situation; 19 from 20 S. aureus cases showed resistance to linezolid. Dr armin stated that as linezolid is not used routinely as a common antibiotic in their hospital, this result was difficult to be accepted but detection of chloramphenicol florfenicol resistant (CFR) positive cases among 15 of 19 strains confirmed that these strains were not only resistant to Linezolid (LZD) but also to $16 \mathrm{mem}$ bered ring macrolides.

Multinational and multicenter surveillance studies reported linezolid susceptibility in more than $99 \%$ of coagulase negative staphylococci and S. aureus clinical strains. Therefore, the author proposes the possibility of this gene transmission from veterinary isolates, such as Staphylococcus sciuri, Staphylococcus warneri, S. aureus, Staphylococcus hyicus or Enterococci (14) as stated by Arias, 2008 (19).

This study indicates the hazard of antibiotic prescription in outpatient cases without obvious indication, and importance of veterinary isolates control, with potential transmission of specific resistance genes to staphylococcal strains in hospital admitted patients.

\section{References}

1. Rammelkamp CH, Maxon T. Resistance of Staphylococcus aureus to the action of penicillin. Experiment Biol Med. 1942;51(3):386-9. doi: 10.3181/00379727-51-13986.

2. Bondi A, Dietz CC. Penicillin Resistant Staphylococci. Experiment Biol Med.1945;60(1):55-8. doi:10.3181/00379727-60-15089. 
3. Barrett FF, Casey JI, Wilcox C, Finland M. Bacteriophage types and antibiotic susceptibility of Staphylococcus aureus. Boston City Hospital, 1967. Arch Intern Med.1970;125(5):867-73. [PubMed: 5444315].

4. Askari E, Soleymani F, Arianpoor A, Tabatabai SM, Amini A, Naderinasab M. Epidemiology of mecA-Methicillin Resistant Staphylococcus aureus (MRSA) in Iran: A Systematic Review and Meta-analysis. Iran J Basic Med Sci. 2012;15(5):1010-9. [PubMed: 23493646].

5. Rahimi F, Bouzari M, Katouli M, Pourshafie MR. Antibiotic resistance pattern of methicillin resistant and methicillin sensitive Staphylococcus aureus isolates in Tehran, Iran. Jundishapur J Microbiol. 2013;6(2):144-9.

6. Rezaee MA, Mirkarimi SF, Hasani A, Sheikhalizadeh V, Soroush MH, Abdinia B. Molecular typing of staphylococcus aureus isolated from clinical specimens during an eight-year period (2005-2012) in Tabriz, Iran. Arch Pediatr Infect Dis. 2016;4(2).

7. Ghasemian A, Najar Peerayeh S, Bakhshi B, Mirzaee M. Accessory gene regulator specificity groups among staphylococcus aureus isolated from hospitalized children. Arch Pediatr Infect Dis. 2014;3(2). doi: 10.5812/pedinfect.16096.

8. Ghasemian A, Najar-Peerayeh S, Bakhshi B, Mirzaee M. High prevalence of icaabcd genes responsible for biofilm formation in clinical isolates of staphylococcus aureus from hospitalized children. Arch Pediatr Infect Dis. 2015;3(3).

9. Askarian M, Zeinalzadeh A, Japoni A, Alborzi A, Memish ZA. Prevalence of nasal carriage of methicillin-resistant Staphylococcus aureus and its antibiotic susceptibility pattern in healthcare workers at Namazi Hospital, Shiraz, Iran. Int J Infect Dis. 2009;13(5):241-7. doi: 10.1016/j.ijid.2008.11.026. [PubMed: 19269873].

10. Arianpoor A, Estaji F, Naderinasab M, Askari E. antimicrobial susceptibility pattern of staphylococcus aureus isolates against newly marketed antibiotics: a report from Imam Reza hospital of Mashhad, Iran. Razavi Int J Med. 2015;3(4).

11. Navidinia M, Fallah F, Lajevardi B, Shirdoost M, Jamali J. Epidemiology of methicillin-resistant staphylococcus aureus isolated from health care providers in mofid children hospital. Arch Pediatr Infect
Dis. 2015;3(2). doi: 10.5812/pedinfect.16458.

12. Daghighi Z, Tajbakhsh S, Goudarzi H, Karimi A, Nateghian A. Molecular detection of vana and vanb genes in vancomycin-resistant enterococcus isolated by polymerase chain reaction from the intestines of children admitted to the intensive care units. Arch Pediatr Infect Dis. 2014;3(2). doi: 10.5812/pedinfect.18414.

13. Aligholi M, Emaneini M, Jabalameli F, Shahsavan S, Dabiri H, Sedaght H. Emergence of high-level vancomycin-resistant Staphylococcus aureus in the Imam Khomeini Hospital in Tehran. Med Princ Pract. 2008;17(5):432-4. doi: 10.1159/000141513. [PubMed: 18685289].

14. Armin S, Rouhipour A, Fallah F, Rahbar M, Ebrahimi M. Vancomycin and linezolid resistant staphylococcus in hospitalized children. Arch Pediatr Infect Dis. 2013;1(1):4-8.

15. Pillai MM, Latha R, Sarkar G. Detection of methicillin resistance in Staphylococcus aureus by polymerase chain reaction and conventional methods: a comparative study. J Lab Physicians. 2012;4(2):83-8. doi: 10.4103/0974-2727.105587. [PubMed: 23441000].

16. Tian Y, Li T, Zhu Y, Wang B, Zou X, Li M. Mechanisms of linezolid resistance in staphylococci and enterococci isolated from two teaching hospitals in Shanghai, China. BMC Microbiol. 2014;14:292. doi: 10.1186/s12866-014-0292-5. [PubMed: 25420718].

17. Dibah S, Arzanlou M, Jannati E, Shapouri R. Prevalence and antimicrobial resistance pattern of methicillin resistant Staphylococcus aureus (MRSA) strains isolated from clinical specimens in Ardabil, Iran. Iran J Microbiol. 2014;6(3):163-8. [PubMed: 25870749].

18. Fatholahzadeh B, Emaneini M, Gilbert G, Udo E, Aligholi M, Modarressi $\mathrm{MH}$, et al. Staphylococcal cassette chromosome mec (SCCmec) analysis and antimicrobial susceptibility patterns of methicillinresistant Staphylococcus aureus (MRSA) isolates in Tehran, Iran. Microb Drug Resist. 2008;14(3):217-20. doi: 10.1089/mdr.2008.0822. [PubMed: 18694326].

19. Arias CA, Vallejo M, Reyes J, Panesso D, Moreno J, Castaneda E, et al. Clinical and microbiological aspects of linezolid resistance mediated by the cfr gene encoding a $23 \mathrm{~S}$ rRNA methyltransferase. J Clin Microbiol. 2008;46(3):892-6. doi: 10.1128/JCM.01886-07. [PubMed: 18174304]. 